\title{
The foundation layer of quantitative cardiac PET/ MRI: Attenuation correction. Again
}

\author{
Stephan G. Nekolla, PhD, FESC, ${ }^{a, b}$ and Jorge Cabello, PhD $^{a}$ \\ ${ }^{a}$ Nuklearmedizinische Klinik Und Poliklinik, Klinikum rechts der Isar, Technische Universität \\ München, Munich, Germany \\ ${ }^{b}$ DZKH (Deutsches Zentrum für Herz-Kreislauf-Forschung e.V.), Partner Site Munich Heart \\ Alliance, Munich, Germany
}

Received Jan 22, 2016; accepted Jan 22, 2016

doi: $10.1007 / \mathrm{s} 12350-016-0424-4$

\section{See related article, pp. 839-846}

It is a well-known fact that attenuation correction is the prerequisite of quantification in PET. It is, however, also the (necessarily related, but even more obvious) requirement that a structure-especially one with a significant extent as the heart represents-with homogeneous tracer uptake is depicted precisely like this: homogeneous. This property is one of the confounding factors why cardiac PET is superior to cardiac SPECT even using static imaging. ${ }^{1}$ Although the fraction of attenuation-corrected SPECT examinations is increasing, the overwhelming majority of SPECT scans is performed without this procedure. From a plain technical perspective, the necessary information is relatively simply derived from any technique, which assesses the radio-density of the imaged body. Typically, a CT scan is used for this purpose. However, as the energy of the $\mathrm{x}$-ray photons is anywhere between 80 and $140 \mathrm{keV}$, only a modest extrapolation to the energy of the photons from the radioactive decay (70 to $159 \mathrm{keV}$ ) is needed. For PET/CT, it is more challenging: as the energy of the annihilation photons is $511 \mathrm{keV}$, this extrapolation is a non-trivial task. Fortunately, a relatively simple algorithm based on experimental data can be used with sufficient accuracy, although one should remember that this is still an extrapolation. ${ }^{2}$ But PET/MRI further

Reprint requests: Stephan G. Nekolla, PhD, FESC, Nuklearmedizinische Klinik Und Poliklinik, Klinikum rechts der Isar, Technische UniversitSt Mnnchen, Ismaninger Str. 22, 81675, Munich, Germany; stephan.nekolla@tum.de

J Nucl Cardiol 2017;24:847-50.

$1071-3581 / \$ 34.00$

Copyright (C) 2016 American Society of Nuclear Cardiology. complicates the situation. An extrapolation is not possible anymore as the MRI signal per se does not contain information about the radio-density for photons of any energy whatsoever. Consequently, attenuation correction was considered-and is still in some settings - an obstacle, which can be exacerbated if we consider scatter as it relies on an attenuation map. When we started the development of an algorithm which is now implemented on the vast majority of all commercially available PET/MRI systems, ${ }^{3}$ an important element was our knowledge of how attenuation maps from the days of standalone PET scanners looked like (Figure 1A). The use of rotating Germanium rod sources was timeconsuming and produced-depending on the age of the sources-images of a quality which required a substantial amount of post-processing to reveal the basic structures of the body-typically answering the question: ${ }^{4}$ body or air. Still, data from those scanners provided the solid platform of PET's value in research and clinical use in cardiology, neurology, and oncology. A value which was confirmed by PET/CT systems-but never challenged-although the patient throughput increased almost an order of magnitude due to the rapid CT scan. What happened, however, was a change in visual perception (Figure 1C). As CT images show so much detail especially in high-contrast objects such as bones (and only a few people ever looked at conventional transmission images which never showed bones unless scanning ex vivo specimen for hours, (Figure 1A), there was the anticipation that an MR-based attenuation map should perfectly resemble a CT scan. Fortunately, this is not the case-at least in the thorax, which is of interest here (the head neck area with a proportionally higher content of cortical bone is a different issue). That the CT data are also mapped to the modest spatial resolution of PET and that for the very high energy of the annihilation photons bones actually 

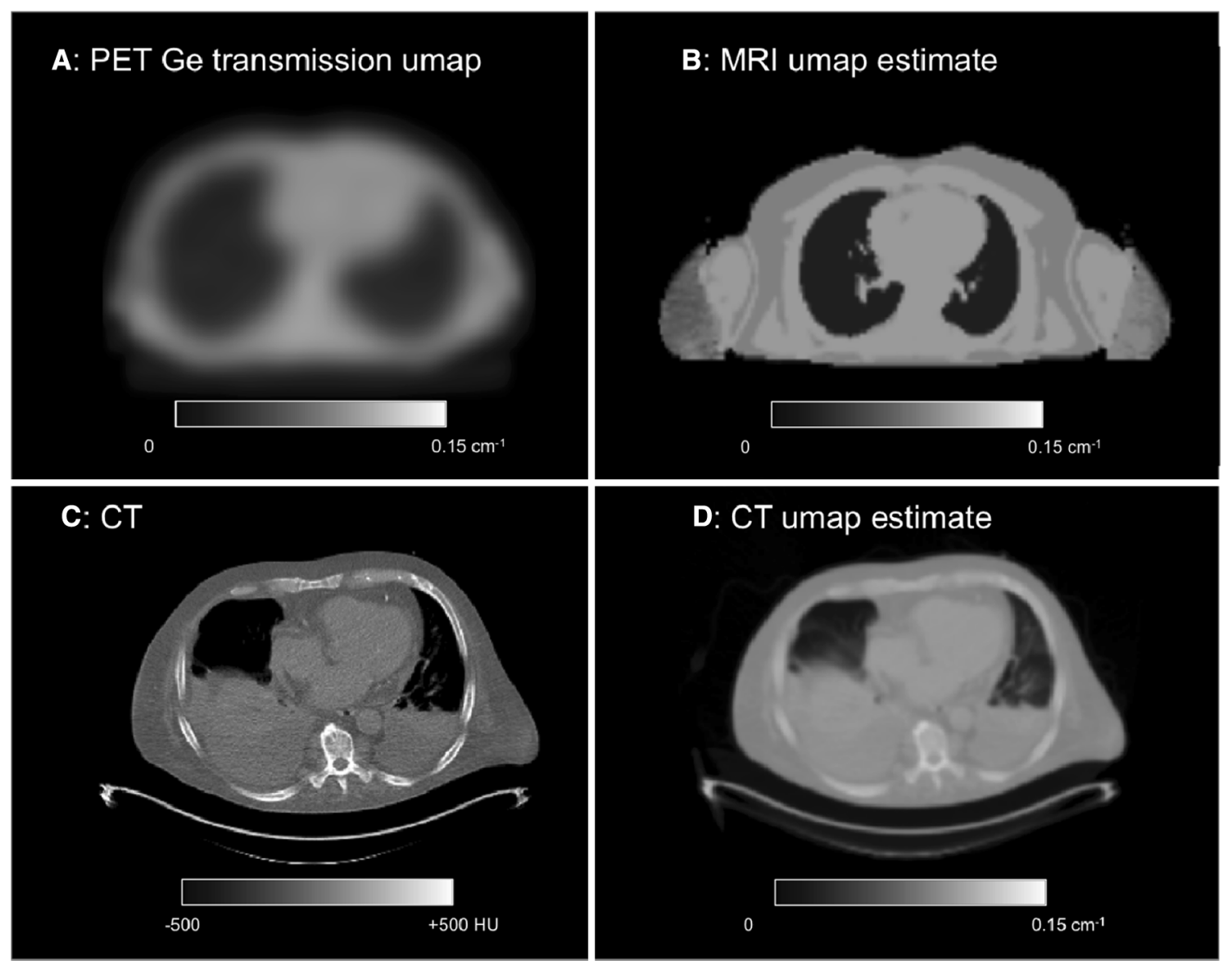

Figure 1. A Transaxial slices through the chest including the heart. Shown are slices from attenuation maps for PET, PET/CT, and PET/MRI. A Transmission map from a conventional PET scanner using rotating germanium rod sources, B MRI-derived attenuation map using a DIXON sequence, $\mathbf{C}$ original CT scan with $120 \mathrm{keV}$, D CT-derived attenuation map (A, B, and $\mathbf{C}$ show different subjects, and $\mathbf{C}$ and $\mathbf{D}$ show the same subject).

appear not fully opaque $\left(\mu_{\text {soft }}\right.$ tissue $=0.096 \mathrm{~cm}^{-1}$ vs $\mu_{\text {bone }}=0.25 \mathrm{~cm}^{-1}$ ), simplifies the matter even more. Thus, the driving force is the soft tissue attenuation. However, especially in obese patients, the contribution of fat can be significant $\left(\mu_{\text {soft tissue }}=0.10 \mathrm{~cm}^{-1}\right.$ vs $\left.\mu_{\text {fat }}=0.086 \mathrm{~cm}^{-1}\right)$. Fortunately, MRI gives quite easy access to a separation between fat and water due to a slightly different resonance frequency from hydrogen protons in fat and water. Thus, the use of a DIXON MRI sequence, which allows this separation, was a natural choice. From a practical point, the acquisition of a volume covering the PET's field-of-view with a reasonable resolution can be accomplished in around $15 \mathrm{~s}$-ideally during a breathhold. Especially in the thorax, the attenuation coefficient from the lung is another factor which needed attention: as the individual value was not known, an average value for the lung was used $\left(\mu_{\text {lung }}=0.018 \mathrm{~cm}^{-1}\right)$. The air-filled cavities of the lungs were identified using means of image processing. This implementation was initially evaluated in oncological patients and yielded a very good agreement. $^{5}$ However, all validation studies following, suffered from the fact that the reference PET/CT scans were acquired prior to the PET/MR examination using FDG, and significant temporal delays were found for logistical reasons. Thus, for physiological reasons, the uptake of FDG is not necessarily constant and this might be especially true if FGD uptake in oncological patients is considered. ${ }^{6}$ But within these limits, studies targeting cardiac tracer uptake as assessed with PET/MRI confirmed these results, although on a per segment level, differences exist. Nensa and colleagues investigated a small cohort of 11 cardiac patients (time p.i. $70 \pm 12$ vs $129 \pm 41$ minutes $),{ }^{7}$ Oldan et al a larger group of oncological patients $(\mathrm{n}=27$, time p.i. $67 \pm 13$ minutes, $\Delta_{\text {Time }}=42 \pm 13$ minutes $)^{8}$ and Vontobel evaluated the approach as implemented on the second commercially available, fully integrated PET/MRI system $(n=23$, oncological patients, $\Delta_{\text {Time }} 46 \pm 17$ minutes). ${ }^{9}$ Lau and colleagues not only confirmed the results using SUV 
values but also investigated the effects of a variable lung signal ( $\mathrm{n}=30$, oncological patients, time p.i. $59 \pm 7$ vs $127 \pm 23$ minutes). ${ }^{10}$ This most recent study confirms the comparability between PET/CT and PET/MR even in the presence of the significant temporal delay between these two scans. Furthermore, the effects of the nonpatient-specific lung attenuation coefficient were found to be not critical for accurate quantification in heart and lesions around the lungs. However, a fixed $\mu_{\text {lung }}$, as used in this PET/MR implementation, has been suggested to be overestimated compared to PET/CT for lung lesions, ${ }^{11}$ and studies extracting patient-specific variable $\mu_{\text {lung }}$ maps from MRI (Turbo-FLASH) were able to reduce quantification errors in volumes of interest in lungs from $12 \%$ to $5 \% .^{12}$

So, where are the remaining precarious factors? As can be seen in Figure 3 in the article from Lau et al, the typical position in a PET/CT is " arms up." This differs from the PET/MR where it is normally "arms down", due to spatial constraints and patient comfort. This fact together with the mismatch of the PET field-of-view of $60 \mathrm{~cm}$ and the MRI field-of-view (FOV) of $45 \mathrm{~cm}$ in the used device creates the problem that the arms and other parts of the body may be outside of the MRI scan's FOV used for the attenuation map. ${ }^{13}$ As one option, the missing tissue can be extrapolated from the non-attenuation-corrected PET data, if there is enough unspecific tracer uptake in these regions ${ }^{14}$-following the simple logic that the presence of tissue is a necessary prerequisite if annihilation events are detected at this location. ${ }^{15}$ This approach was already used in the validation study from Oldan et $\mathrm{al}^{8}$ (Figure 1B). Interestingly, this option was not used in the study by Lau et al and could have contributed to the regional differences observed and also the reduced image quality in their Figure 3.

A final component which has not been investigated so far are the artifacts from metallic compounds such as artificial valves, pacemakers, and other interventional devices in this specific cardiac imaging setting. From PET/CT, it is already known that beam-hardening artifacts from metallic implants can impact the quality of the PET examination. ${ }^{16}$ In MRI, however, the effect is the other way around: conducting materials create signal voids, which clearly extend the true object size. ${ }^{17}$ So far, this is not a real issue in cardiac PET/MRI as the MR compatible devices are typically cleared for 1.5 Tesla and not yet for 3 Tesla as used in the current PET/MRI systems, but this will likely change in the next years.

In summary, the study by Lau et al is a welcomed contribution showing that attenuation correction in cardiac PET/MRI is routinely feasible if appropriate measures of quality control are observed-a statement which, however, holds true for PET/CT as well. ${ }^{18}$

\section{Disclosure}

None.

\section{References}

1. Bateman TM, Heller GV, McGhie AI, Friedman JD, Case JA, Bryngelson JR, et al. Diagnostic accuracy of rest/stress ECG-gated Rb-82 myocardial perfusion PET: Comparison with ECG-gated Tc-99 m sestamibi SPECT. J Nucl Cardiol 2006;13:24-33.

2. Kinahan PE, Townsend DW, Beyer T, Sashin D. Attenuation correction for a combined 3D PET/CT scanner. Med Phys 1998;25:2046-53.

3. Martinez-Moller A, Souvatzoglou M, Delso G, Bundschuh RA, Chefd'hotel C, Ziegler SI, et al. Tissue classification as a potential approach for attenuation correction in whole-body PET/MRI: Evaluation with PET/CT data. J Nucl Med 2009;50:520-6.

4. Meikle SR, Dahlbom M, Cherry SR. Attenuation correction using count-limited transmission data in positron emission tomography. J Nucl Med 1993;34:143-50.

5. Drzezga A, Souvatzoglou M, Eiber M, Beer AJ, Fürst S, MartinezMöller A, et al. First clinical experience with integrated wholebody PET/MR: Comparison to PET/CT in patients with oncologic diagnoses. J Nucl Med 2012;53:845-55.

6. Inglese E, Leva L, Matheoud R, Sacchetti G, Secco C, Gandolfo P, et al. Spatial and temporal heterogeneity of regional myocardial uptake in patients without heart disease under fasting conditions on repeated whole-body 18F-FDG PET/CT. J Nucl Med 2007; 48:1662-9.

7. Nensa F, Poeppel TD, Beiderwellen K, Schelhorn J, Mahabadi AA, Erbel R, et al. Hybrid PET/MR Imaging of the heart: Feasibility and initial results. Radiology 2013;268:366-73.

8. Oldan JD, Shah SN, Brunken RC, DiFilippo FP, Obuchowski NA, Bolen MA. Do myocardial PET-MR and PET-CT FDG images provide comparable information? J Nucl Cardiol 2015. doi: 10.1007/s12350-015-0159-7.

9. Vontobel J, Liga R, Possner M, Clerc OF, Mikulicic F, VeitHaibach P, et al. MR-based attenuation correction for cardiac FDG PET on a hybrid PET/MRI scanner: Comparison with standard CT attenuation correction. Eur J Nucl Med Mol Imaging 2015;42: 1574-80.

10. Lau MCJ, Laforest R, Sotoudeh H, Nie X, Sharma S, McConathy $\mathrm{J}$, et al. Evaluation of attenuation correction in cardiac PET using PET/MR. J Nucl Cardiol 2016. doi:10.1007/s12350-015-0197-1.

11. Rauscher I, Eiber M, Furst S, Souvatzoglou M, Nekolla SG, Ziegler SI, et al. PET/MR imaging in the detection and characterization of pulmonary lesions: Technical and diagnostic evaluation in comparison to PET/CT. J Nucl Med 2014;55:724-9.

12. Marshall HR, Prato FS, Deans L, Theberge J, Thompson RT, Stodilka RZ. Variable lung density consideration in attenuation correction of whole-body PET/MRI. J Nucl Med 2012;53:977-84.

13. Delso G, Martinez-Moller A, Bundschuh RA, Nekolla SG, Ziegler SI. The effect of limited MR field of view in MR/PET attenuation correction. Med Phys 2010;37:2804-12.

14. Nuyts J, Bal G, Kehren F, Fenchel M, Michel C, Watson C. Completion of a truncated attenuation image from the attenuated PET emission data. IEEE Trans Med Imaging 2013;32:237-46. 
15. Martinez-Moller A, Souvatzoglou M, Navab N, Schwaiger M, Nekolla SG. Artifacts from misaligned CT in cardiac perfusion PET/CT studies: Frequency, effects, and potential solutions. J Nucl Med 2007;48:188-93.

16. DiFilippo FP, Brunken RC. Do implanted pacemaker leads and ICD leads cause metal-related artifact in cardiac PET/CT? J Nucl Med 2005;46:436-43.
17. Buchbender C, Hartung-Knemeyer V, Forsting M, Antoch G, Heusner TA. Positron emission tomography (PET) attenuation correction artefacts in PET/CT and PET/MRI. Br J Radiol 2013;86:20120570.

18. Nekolla SG, Martinez-Moller A. Attenuation correction in cardiac PET: To raise awareness for a problem which is as old as PET/CT. J Nucl Cardiol 2015;22:1296-9. 